\title{
Visualization of Thrombosis on a Prosthetic Pulmonary Valve Using Electrocardiogram- Gated Multidetector Computed Tomography
}

\author{
Shuhei Kakizaki ${ }^{1}$ Seiichi Tazawa ${ }^{1}$ Shigeo Kure ${ }^{1}$ Yoshikatsu Saiki ${ }^{2}$ \\ ${ }^{1}$ Department of Pediatrics, Tohoku University School of Medicine, \\ Sendai, Japan \\ 2 Department of Cardiovascular Surgery, Tohoku University School of \\ Medicine, Sendai, Japan

\begin{abstract}
Address for correspondence Shuhei Kakizaki, MD, PhD, Department of Pediatrics, Tohoku University School of Medicine, 1-1 Seiryo-machi Aoba-ku, Sendai 980-8574, Japan (e-mail: skakizaki@med.tohoku.ac.jp).
\end{abstract}

Thorac Cardiovasc Surg Rep 2014;3:6-8.

\begin{abstract}
Keywords

- stuck valve

- computed tomography

- thrombolysis

We report a case of prosthetic valve thrombosis in an 18-year-old man. Total surgical repair of tetralogy of Fallot was performed at 3 years of age. At the age of 18, he underwent pulmonary valve replacement because of exacerbating pulmonary trunk obstruction. After surgery, valve malfunction was suspected. We attempted to employ electrocardiogram (ECG)-gated multidetector computed tomography (MDCT). The stuck valve was clearly visualized together with a thrombus within. Thrombolytic therapy was undertaken on these findings. ECG-gated MDCT allowed us to make a definitive diagnosis of valve thrombosis as the cause of valve malfunction, which led us to avoid surgical reintervention.
\end{abstract}

\section{Case Description}

The patient was an 18-year-old man with Down syndrome and tetralogy of Fallot. At the age of 3 years, he underwent surgical reconstruction of the right ventricular outflow tract with a porcine pericardial monocusp patch and closure of a ventricular septal defect. Right ventricular outflow tract obstruction progressed in the late postoperative phase. Cardiac catheterization performed at the age of 17 years demonstrated a pressure gradient of $80 \mathrm{~mm} \mathrm{Hg}$ between the right ventricle and pulmonary artery and a right-to-left ventricular systolic pressure ratio of 0.82 . Calcification of the pulmonary valve was also noted at this time. Consequently, pulmonary valve replacement surgery was performed at the age of 18 years, and a bileaflet mechanical prosthetic valve (SJM valve; St. Jude Medical Inc., Little Canada, Minnesota, United States) was implanted. Continuous heparin infusion was commenced postoperatively on day 1 , and oral administration of warfarin was started on day 3. However, on postoperative day 6 , pulmonary regurgitation was noted on echocardiography, and malfunction of the mechanical valve was suspected.
A cine-fluoroscopic examination of the mechanical valve showed that the valve was stuck in the systolic position during systole and diastole ( - Fig. 1a). Electrocardiogram (ECG)-gated multidetector computed tomography (MDCT) (Somatom Definition; Siemens Medical Solutions, Forchheim, Germany) was immediately performed, which revealed a filling defect extending across the two leaflets of the bileaflet valve ( $\mathbf{- F i g} \mathbf{1 b} \mathbf{1 b}$ ). Furthermore, an immobile thrombus around the hinge on the left side was clearly and distinctively visualized on cine-multiplanar reformatting (cine-MPR) throughout a cardiac cycle (-Fig. 1c), and valve thrombosis was diagnosed as the cause of the patient's valve malfunction. He was subsequently administered intravenous mutant tissue plasminogen activator (monteplase; Cleactor; Eisai Co Ltd, Tokyo, Japan) at a dose of $14,000 \mathrm{IU} / \mathrm{kg}$. On examination 1 hour after monteplase administration, mechanical valve sounds could be heard once again. In addition, a repeat cine-fluoroscopic examination showed good and symmetrical opening of the bileaflet valve, and disappearance of the thrombus was also later confirmed on cine-MPR (-Figs. 2a, b, and 3a-f). No complications such as hemorrhage were noted following monteplase administration.
License terms

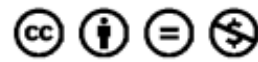

received

October 4, 2013 accepted after revision

December 23, 2013

published online

March 26, 2014
DOI http://dx.doi.org/

10.1055/s-0034-1371392. ISSN 2194-7635. (c) 2014 Georg Thieme Verlag KG Stuttgart · New York 


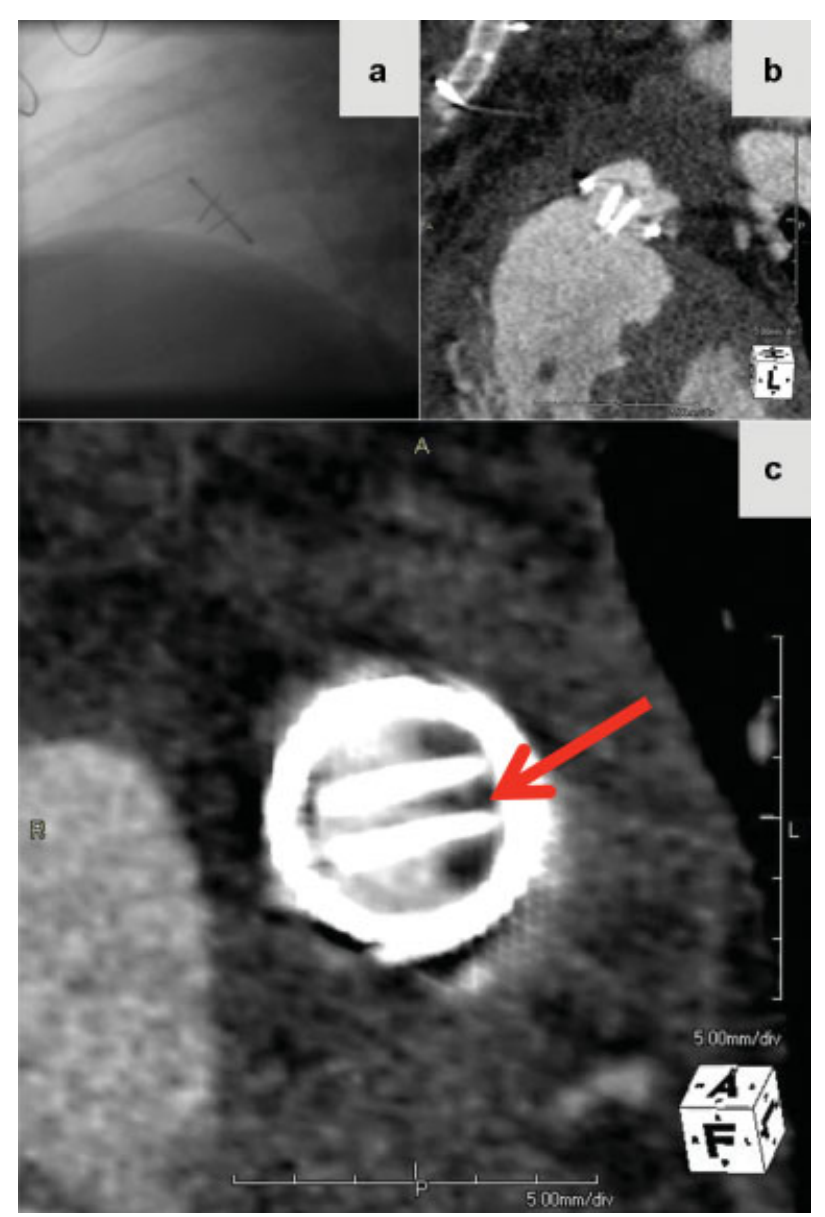

Fig. 1 Cine-fluoroscopic examination of the valve showing that the valve is stuck in the systolic position (a). However, the causative mechanism was not identified in this study. There are almost no metallic artifacts generated by the mechanical valve on cine-multiplanar reformatting (cine-MPR) (b). Cine-MPR showing the filling defects in the cross-sectional image of the mechanical valve. The thrombosis was diagnosed on the basis of the filling defect noted on the left side of the mechanical valve (red arrow) (c).

\section{Discussion}

Transthoracic echocardiography is one of the most common noninvasive examinations used to diagnose prosthetic valve dysfunction. However, significant imaging artifacts are often encountered in patients with metallic prostheses. Transesophageal echocardiography (TEE) has been reported as an alternative. ${ }^{1}$ However, even with TEE, the echo beam is interrupted by the metallic component. Furthermore, the anterior location of the pulmonary valve makes visualization of the prosthetic valve even more difficult with TEE. ECGgated MDCT enables rapid scanning and provides superior temporal and spatial resolution with fewer artifacts through the use of multiple detector rows. In this case, ECG-gated MDCT was able to demonstrate the presence of a thrombus formed in the mechanical pulmonary valve that extended across both leaflets ( - Fig. 1c). In addition, cine-MPR showed the bileaflet valve stuck in the systolic position (-Fig. 1b, c). On imaging, there were almost no metallic artifacts generated by the mechanical valve. Thus, we obtained visualization of the thrombus and a definitive diagnosis of prosthetic valve thrombosis.

The patient had chosen a mechanical prosthetic valve for the correction of right ventricular outflow tract obstruction. At present, there is controversy over the selection of biological versus mechanical prostheses for the reconstruction of right ventricular outflow tract. ${ }^{2}$ Although mechanical valves are superior to biological valves in terms of durability, they are associated with a risk of thrombus formation; thus, anticoagulant therapy is usually recommended after surgery. ${ }^{3}$ Unfortunately, thrombosis occurred in this patient despite the administration of anticoagulant therapy in the early postoperative period. In patients with prosthetic pulmonary valve, the risk of valve thrombosis is highest in the presence of low cardiac output in the early postoperative period. ${ }^{4}$ As such, the anticoagulant therapy by the patient in this case was possibly inadequate to prevent thrombus formation.

Thrombolytic therapy has been reported to be effective for the treatment of prosthetic valve thrombosis for patients

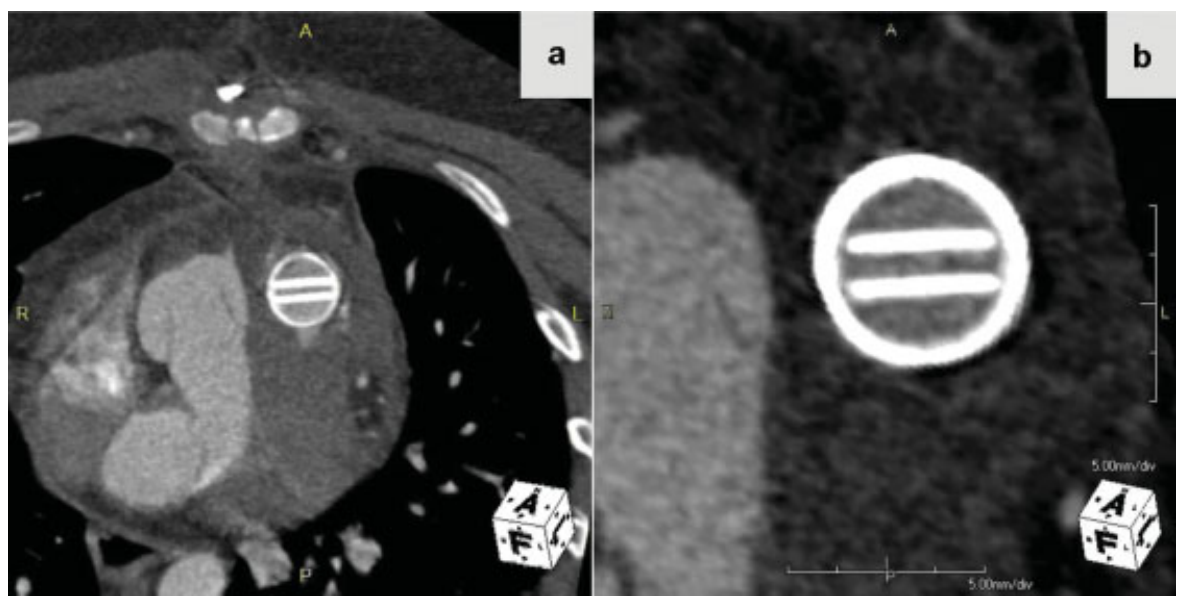

Fig. 2 Cine-multiplanar reformatting (cine-MPR) confirmed the disappearance of the filling defect. Cine-MPR showing the cross-sectional image of the mechanical valve (a) and magnified view (b). 


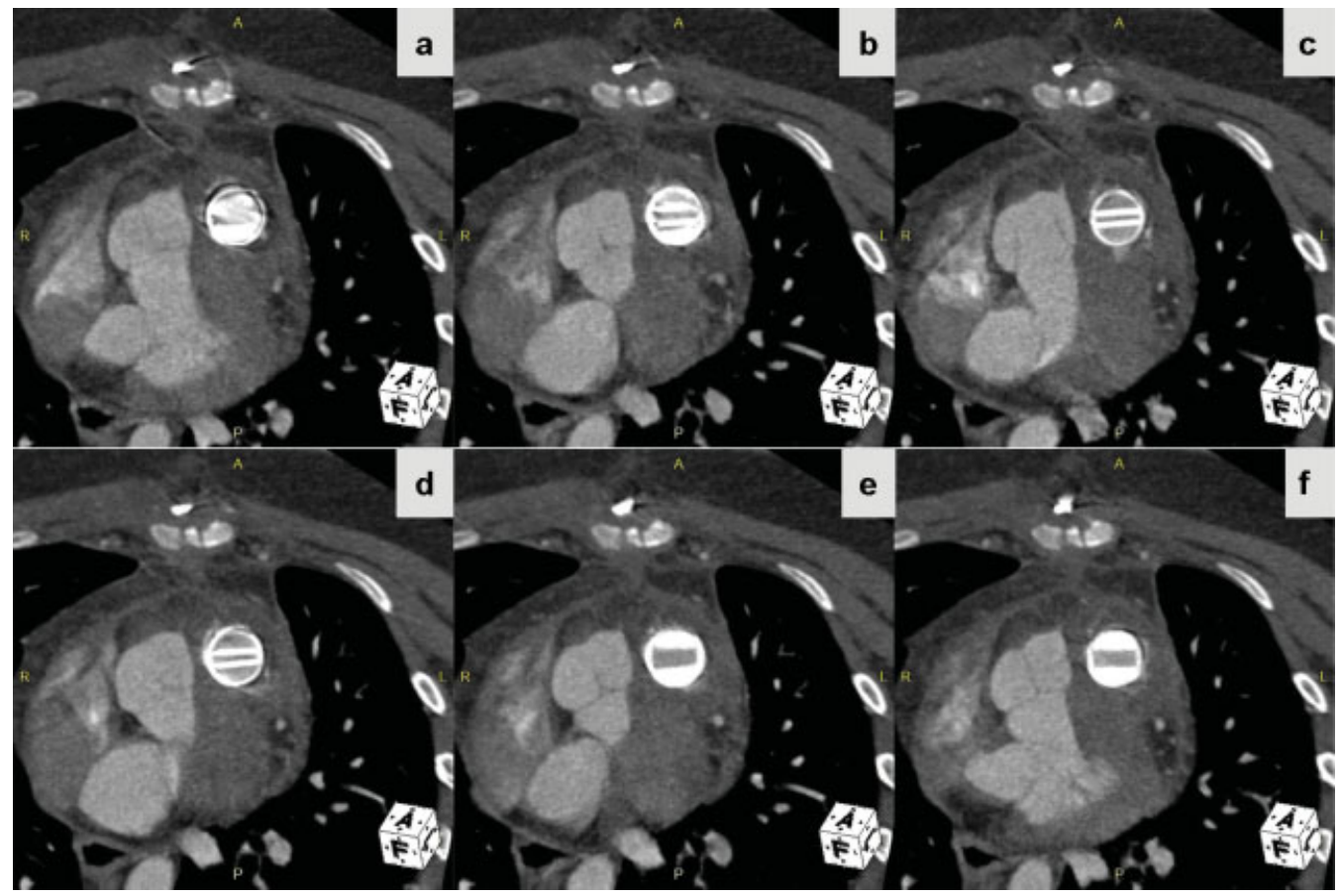

Fig. 3 Maximal excursion of the leaflets is demonstrated by electrocardiogram-gated multidetector computed tomography after a successful thrombolytic therapy. Cine-multiplanar reformatting showed free mobility of both leaflets in the systolic phase $(a-c)$ and in the diastolic phase (d-f).

without contraindications for thrombolysis. ${ }^{5}$ In this case, we choose monteplase for thrombolytic therapy. Monteplase, a genetic recombinant tissue-type plasminogen activator, is highly effective for acute pulmonary embolism and offers acceptable safety for severe bleeding complications. ${ }^{6}$

The patient was treated successfully with thrombolytic therapy and showed clinical improvement as evidenced by the resumption of valvular click sound within approximately 1 hour after treatment. Complications such as hemorrhage and pulmonary embolism were not observed. Because valve re-replacement after valve malfunction is associated with significant risks, ${ }^{7}$ we were fortunate to not have to proceed to surgical reintervention in this patient. ECG-gated MDCT led to prompt administration of thrombolytic agents.

We also found ECG-gated MDCT performed after thrombolytic therapy to be useful in assessing the effectiveness of thrombolytic therapy (-Figs. 2a, b, and 3a-f). In this case, it was repeated to confirm the disappearance of the thrombus and evaluate valve function following thrombolysis. Residual thrombus was ruled out by this follow-up examination. Further treatment was not required on the basis of these findings. ECG-gated MDCT is a powerful diagnostic modality for mechanical valvular thrombosis.

\section{References}

1 Cáceres-Lóriga FM, Pérez-López H, Santos-Gracia J, Morlans-Hernandez K. Prosthetic heart valve thrombosis: pathogenesis, diagnosis and management. Int J Cardiol 2006;110(1):1-6

2 Husain SA, Brown J. When reconstruction fails or is not feasible: valve replacement options in the pediatric population. Semin Thorac Cardiovasc Surg Pediatr Card Surg Annu 2007:117-124

3 Nurozler F, Bradley SM. St. Jude medical valve in pulmonary position: anticoagulation and thrombosis. Asian Cardiovasc Thorac Ann 2002;10(2):181-183

4 Butchart EG, Gohlke-Bärwolf C, Antunes MJ, et al; Working Groups on Valvular Heart Disease, Thrombosis, and Cardiac Rehabilitation and Exercise Physiology, European Society of Cardiology. Recommendations for the management of patients after heart valve surgery. Eur Heart J 2005;26(22):2463-2471

5 Cáceres-Lóriga FM, Pérez-López H, Morlans-Hernández K, et al. Thrombolysis as first choice therapy in prosthetic heart valve thrombosis. A study of 68 patients. J Thromb Thrombolysis 2006;21(2):185-190

6 Niwa A, Nakamura M, Harada N, Musha T. Observational investigation of thrombolysis with the tissue-type plasminogen activator monteplase for acute pulmonary embolism in Japan. Circ J 2012; 76(10):2471-2480

7 Giulio R, Tomaso B, Lorenza P, Domenico S, Gaetano T, Dino C. Multivariate analysis of survival after malfunctioning biological and mechanical prosthesis replacement. Ann Thorac Surg 1998; 66:88-94 